\title{
A Novel Approach for Managing Channels in Wireless Network
}

\author{
Ram Krishan \\ Department of Computer Science \\ Punjabi University Guru Kashi College \\ Talwandi Sabo-Bathinda(Punjab) \\ Sukhwinder Singh \\ Yadavindra College of Engineering \\ Punjabi University Guru kashi Campus \\ Talwandi Sabo-Bathinda(Punjab) \\ Sukhwinder Singh \\ Department of Computer Science \\ Punjabi University Guru Kashi College \\ Talwandi Sabo-Bathinda(Punjab)
}

\begin{abstract}
In this paper, we propose a Channel Management Algorithm at the Access Points (APs) of Wireless Local Area Network (WLAN) in order to maximize the efficiency of the network by managing users on different channels such that the overall throughput is maximized. We start with the channel assignment at the APs, which is based on minimizing the total interference between APs. Initially we assign the users at different APs on the basis of the users load. The solution developed in this paper uses a distributed algorithm by assigning non-overlapping channels and managing fixed and roaming users in network. One software agent manages all APs and is able to communicate with its neighbors in order to maximize channel utilization for global throughput. Proposed algorithm result shows efficient management of users on different APs thus increases the channel utilization in WLAN. It also improves the network performance and gives higher throughput.
\end{abstract}

\section{Categories and Subject Descriptors}

C.2.1 [Wireless Networks]: Network Architecture and Design Wireless communication.

\section{General Terms}

Algorithms, Management, Performance.

\section{Keywords}

WLAN, Channel Management, Access Point, Channel Interference.

\section{INTRODUCTION}

Channel assignment in IEEE 802.11 WLAN has received significant attention in the past few years [1]. The 802.11 wireless LAN operate in the unlicensed ISM frequency, and all access points share the same frequency. The increase in deployment of access points (APs) has led researchers to develop channel assignment algorithms in order to reduce co-channel and adjacent channel interferences from neighboring APs, which causes an overall throughput degradation of the network.

Efficient assignment of channels becomes necessary to avoid and minimize interference. Roaming is the ability of a user to seamlessly switch between access points while moving or for load balancing [7] purposes. The user should associate [8] with the access point with the strongest signal. To do this APs need to be on the same subnet (to avoid needing to acquire a new IP address) and have the same SSID and encryption keys [3]. The proposed algorithm is used for channel management in WLAN. The objective of proposed work is to manage the channels in order to maximize the channel utilization [9], minimize the network traffic and to increase the network throughput by users load distribution. This results in a higher throughput and better performance of the network.

In this paper, we have presented algorithm which manages the network by assigning non-interfering channels to all APs and also provides the better channel utilization with roaming users. Firstly we have done the channel assignment that minimizes channel interference [2] between neighboring APs. In next step we manage the users on APs by applying our algorithm so that the network throughput increases. The result of our algorithm gives the better channel management and also increases the network throughput.

\section{CHANNEL INTERFERENCE}

In IEEE $802.11 \mathrm{~b} / \mathrm{g}$ WLAN, there exist 14 channels. Channels 1,6 and 11 are non-overlapping [2], as shown in Fig. 1. Shows the 14 channels, only 11 are used in the US. Each channel spreads over $22 \mathrm{MHz}$ due to the Direct Sequence Spread Spectrum (DSSS) technique employed by IEEE $802.11 \mathrm{~b} / \mathrm{g}$.

For instance, channel 1 range is from $2401 \mathrm{GHz}$ to $2.423 \mathrm{GHz}$ and its center frequency is $2.412 \mathrm{GHz}$ [4]. The center frequency of two adjacent channels

is separated by $5 \mathrm{MHz}$. Therefore, there is channel bandwidth overlap [6]. The interference-level factor $w j k$ is defined as follows

$W_{a b}=\max \left(0,1-\left|C h_{a}-C h_{b}\right| \mathbf{x} c\right) \quad \ldots . .(1)$

Where $C_{a}$ is the channel assigned to $A P_{a}, C h_{b}$ is the channel assigned to $A P_{b}$ and $c$ is the non-overlapping portion of two adjacent channels, expressed as a fraction of the frequency spectrum of channel. For instance, channel 1 and channel 2 do not overlap from $2.401 \mathrm{GHz}$ to $2.406 \mathrm{GHz}$, as shown in Fig. 1. Normalizing the overlap of $5 \mathrm{MHz}$ over the spectrum of $23 \mathrm{MHz}$, $c$ is equal to $1 / 5$ approximately. When the channels are far apart, as is the case with channels 1 and $6, w_{a b}=0$ (i.e., no interference). When the two channels are the same, $C h_{a}-C h_{b}=0$, Eqn 1 suggests that $w_{a b}=1$ (i.e., maximum interference). 


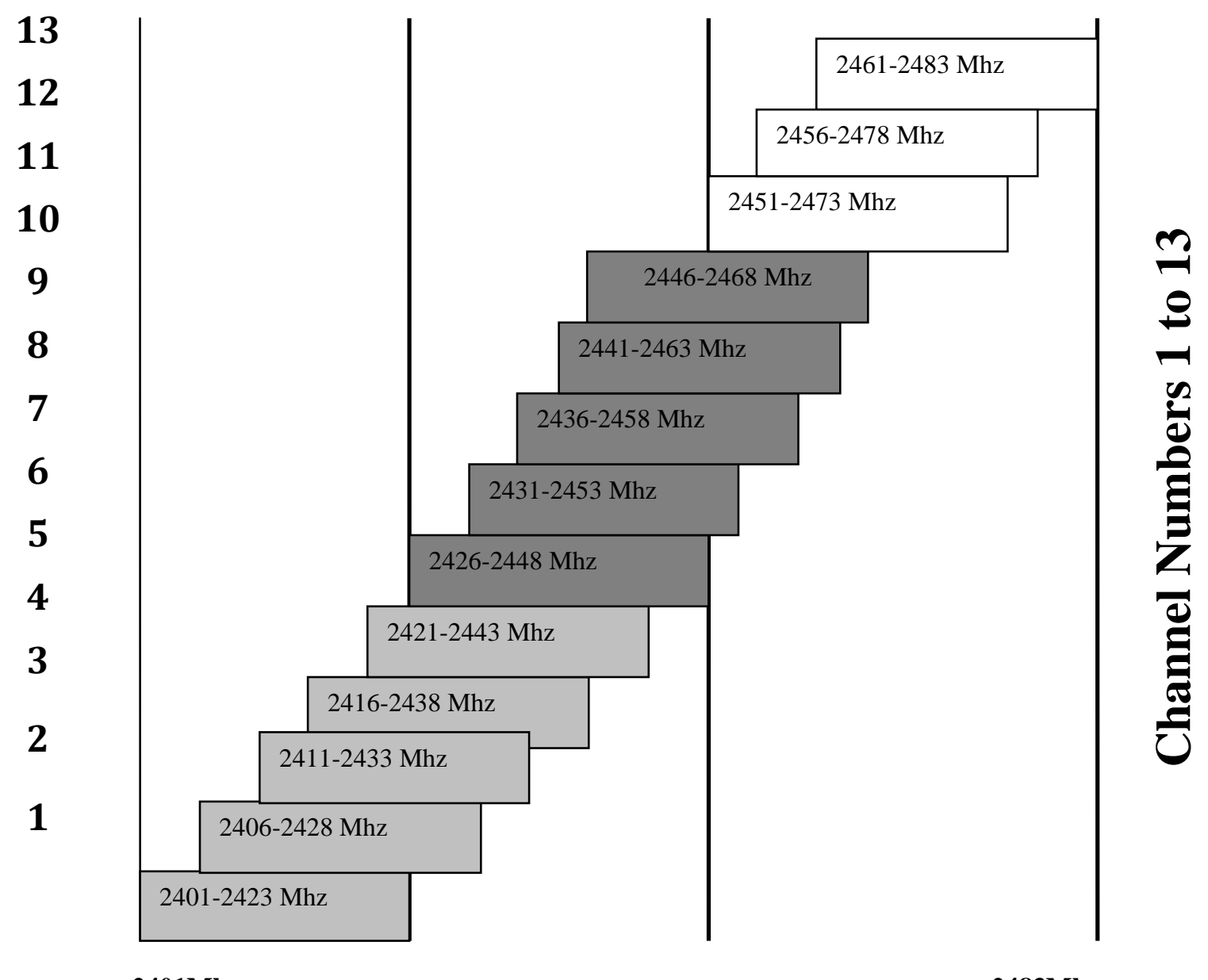

FREQUENCY

Figure1. Frequency Spread-spectrum of $802.11 \mathrm{~b} / \mathrm{g}$ with three non-overlapping Channels

Therefore, channels should be assigned to APs such that overlapping channel interference is minimized. On the other hand, for channels 1 and $6,\left|C h_{a}-C h_{b}\right|=5, w_{a b}=0$, suggesting no interference. Mindful that we only have limited channel resources (11 channels in IEEE $802.11 \mathrm{~b} / \mathrm{g}$ ), some channels need to be "recycled." If the same channel is to be assigned to two or more APs which are located far enough from each others, the overlapping channel interference signal detected by each AP should be less than a given threshold.

\section{CHANNEL ASSIGNMENT MODEL}

Here, we will present a new channel assignment model and algorithm for IEEE 802.11 WLAN systems. Channels should be assigned to each AP in such a way to maximize the throughput of network at user level, rather than to minimize interference among APs. The algorithm in [5] deals with distributing the load more efficiently among APs by reassigning users to different APs by switching users to the Least Congested AP (LCAP). By distributing user on APs, the network channels will be utilizedmore efficiently which results the higher throughput. We consider a WLAN consisting of $M$ APs situated in an indoor single-floor service area [10]. A set of randomly distributed $N$ users are to be served by these APs. Our algorithm is initiated by balancing the load on each AP on basis of the users assignment at least congested AP [6]. Each user in network can access the number of

APs but a user is assigned to one and only one AP at any time. When a user is having the access of more than one AP then the user [13] can check the traffic on available APs and can associate with any of the available AP.

\subsection{Range Set of User}

For each user we define its range set [7] as the set of all APs such that the user lies within the communication range of each such AP, regardless of the current channel of operation of the APs. In Figure 2, the range set for U4 is (AP1 and AP3) and for U7 is (AP2 and AP3). Note that a user can compute its range set empirically by monitoring APs in its vicinity and has to associate to one of such APs to obtain network service. 


\subsection{Interference Set of User}

The range set of users captures some of the interference [7] experienced by the users, but does not capture the total interference observed by the user-AP link. A user can suffer additional interference from users of other APs, if the user is within the transmission range of such users. Note that if the client is within the transmission range of such an AP, then the AP will be in the range set. On the other hand, if the client is outside the transmission range of such an AP, the latter becomes a part of the interference set. In real time user join or leaves network regularly, results traffic on APs is increases or decreases so user can switch from one channel to another available channel. User switches decreases the network traffic [12] which gives the efficient channel management and also increases the overall performance of network.
The above algorithm firstly assigns the non-overlapping channels to the N APs and User Load balancing on APs is done by checking users on each AP. The user and AP association in network is not fixed because the user enters or leaves the network. This algorithm can ensure efficient operation of a WLAN as the user enters or leaves in network [11]. To test this hypothesis, a simulation is run continuously in the network and it manages the traffic on channels. Users load [14] on different APs is managed by switching users to the other available AP which is in the Range Set of user thus manages the channels.

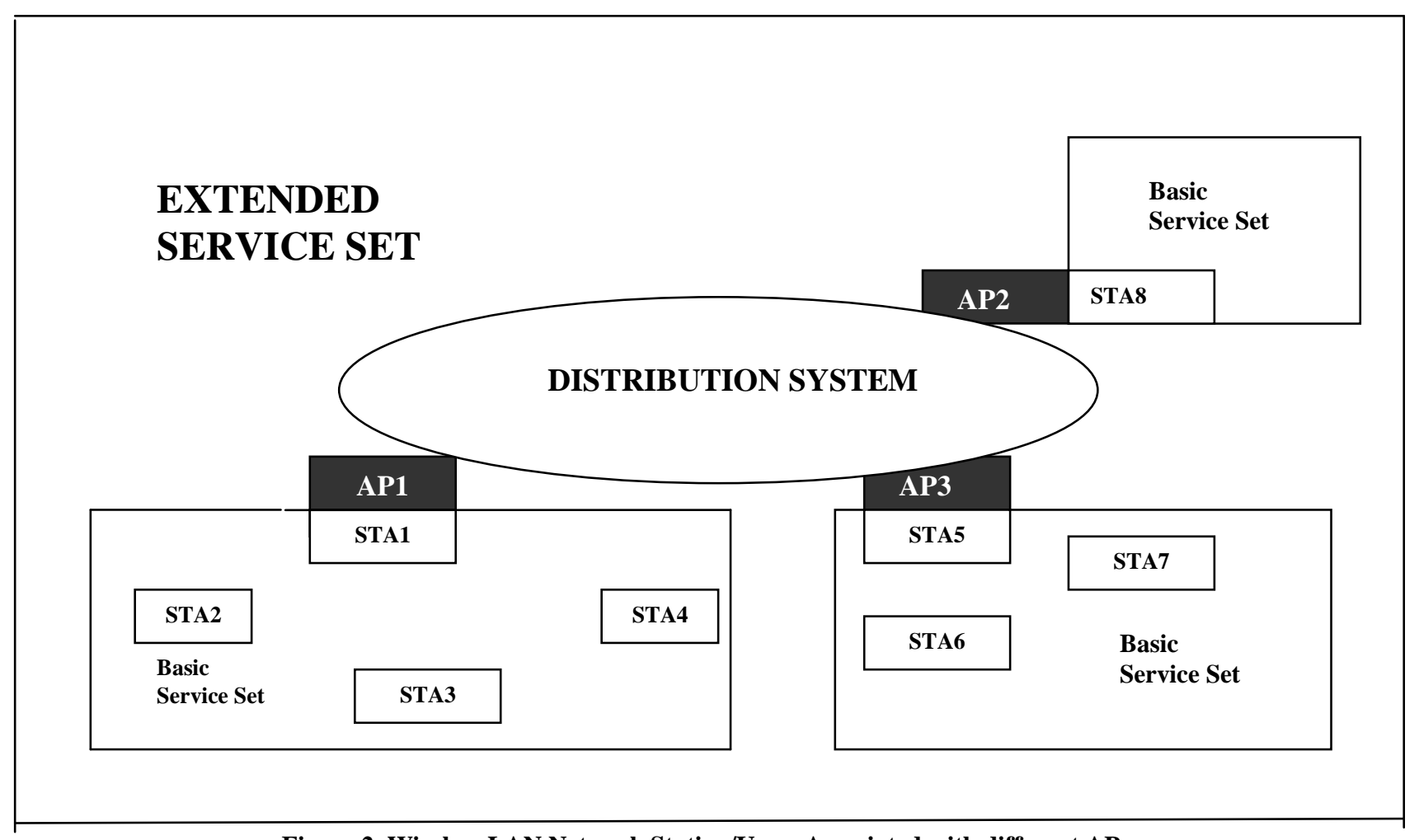

Figure 2. Wireless LAN Network Station/Users Associated with different APs.

\section{CHANNEL MANAGEMENT ALGORITHM}

I. Assign channels to the $N$ APs based on the NLIP model proposed in reference [6] which is based on minimizing the total interference between APs.

II. Input the users in network.

III. Perform load balancing based on user's traffic.

IV. Compute the network traffic as the user enters or leaves the network.

V. Switch users between APs to manage traffic on channels.

VI. Compute the network traffic after a time slice and go back to step 4.

\section{ALGORITHM RESULTS}

The simulations were carried out with service areas consisting of 4 APs and 10, 15, 20, 25, 30, 40 and 50 users, respectively, forming a WLAN. As the new user enters in the network it associates with the AP which is in the Range Set of user and having least congested (LCAP). Initial assignment of channels to various APs is fixed. We have shown the simulation results at different time intervals and with moving user in the network.

\subsection{Simulation Scenario 1}

In Scenario 1, we have 5 APs and 5 users. Figure 3 (a) shows the initial association of the users with different APs on the basis of the range set of users given in the right side of the table. Figure 
3(b) shows the final result of the user distribution on APs after running our algorithm. If we look closer to the range set of the users we will notice that the user having range of more than one AP can associate to either AP on particular channel which is assigned to the AP.

\subsubsection{Range Set}

Range Set of U1 (AP1, AP2)

Range Set of U2 (AP1, AP2,AP5)

Range Set of U3 (AP4,AP2,AP1)
Range Set of U4 (AP1,AP2,AP3)

Range Set of U5 (AP1,AP2)

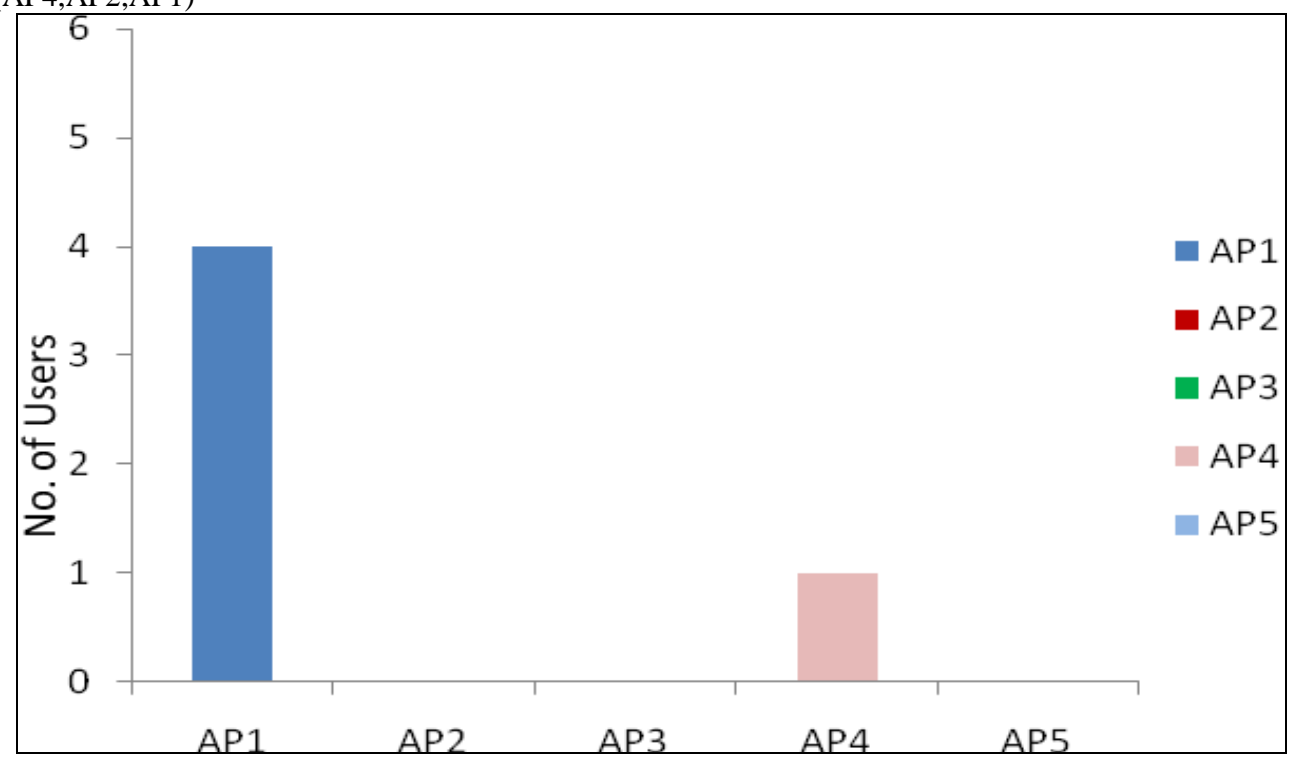

Figure 3(a). Initial User AP Association

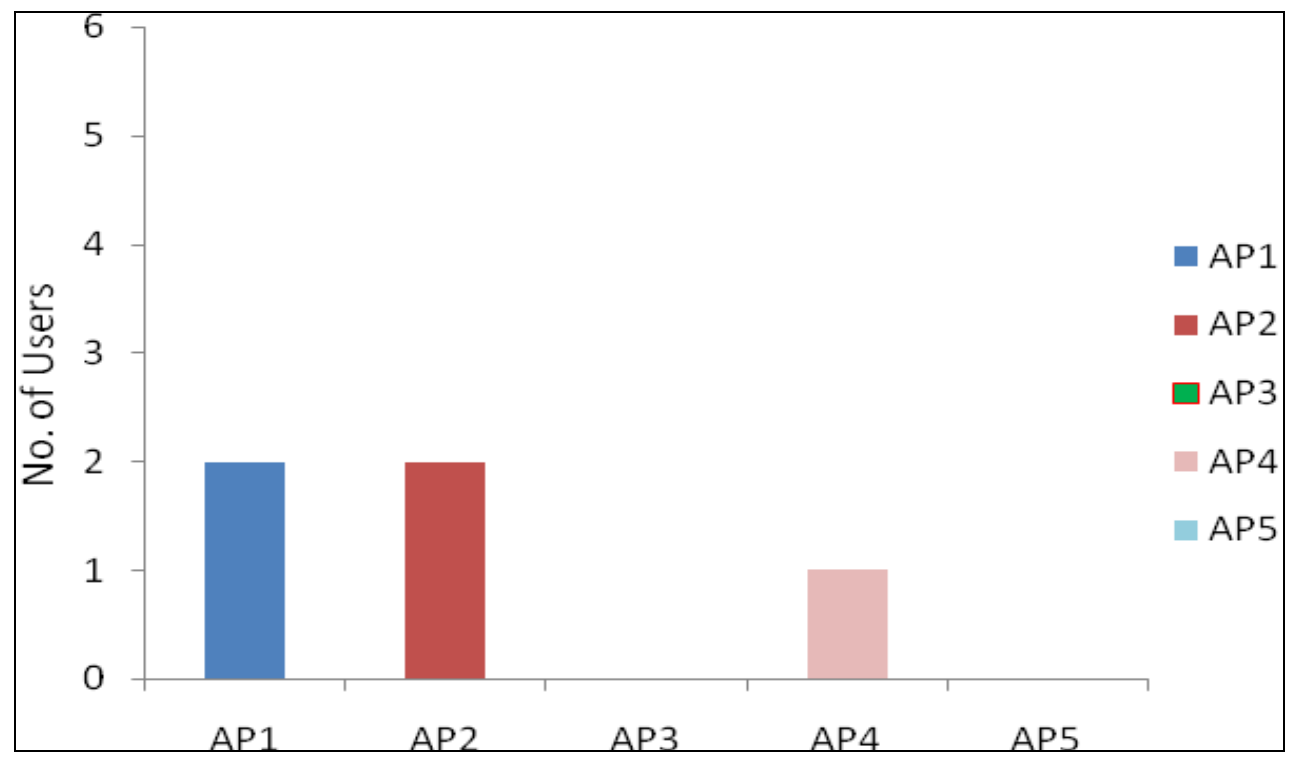

Figure 3(b). Finial User AP Association

In the above figures we have shown the comparison of the initial user distribution on APs and distribution of users after running our algorithm. We notice that the two users are switched from AP1 to AP2 thus managing the load on respective channels. Our algorithm runs for each user in network to check best possible association with AP which leads to better load distribution and channel management. 


\subsection{Simulation Scenario 2}

In Scenario 2, we have 5 APs and 15 users. Figure 4 (b) shows the final result of the user distribution on APs after running our algorithm.

5.2.1 Range Set

Range Set of U1 (AP1, AP2)

Range Set of U2 (AP1)

Range Set of U3 (AP4)

Range Set of U4 (AP1)

Range Set of U5 (AP1, AP2)
Range Set of U6 (AP3)

Range Set of U7 (AP4, AP5)

Range Set of U8 (AP5, AP2)

Range Set of U9 (AP3)

Range Set of U10 (AP4)

Range Set of U11 (AP3)

Range Set of U12 (AP3, AP5)

Range Set of U13 (AP3, AP4)

Range Set of U14 (AP5)

Range Set of U15 (AP3, AP5)

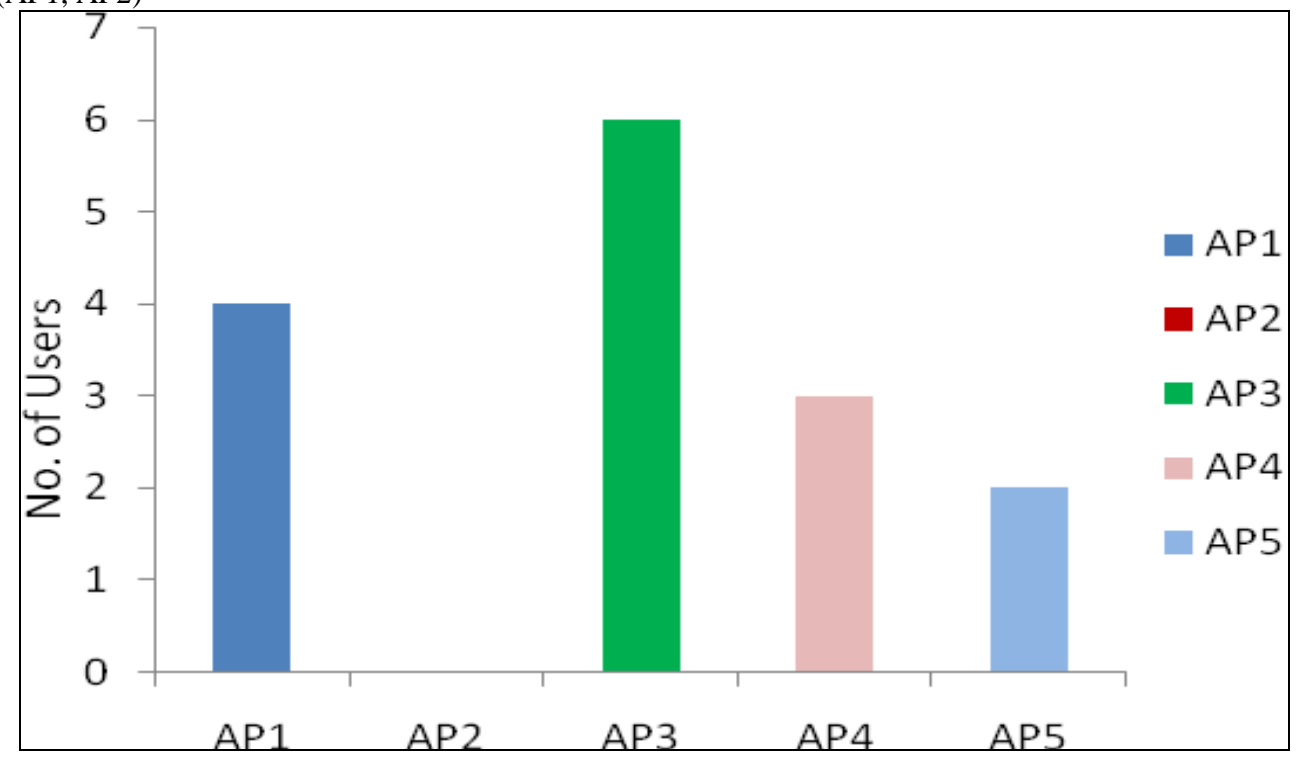

Figure 4(a). Initial User AP Association

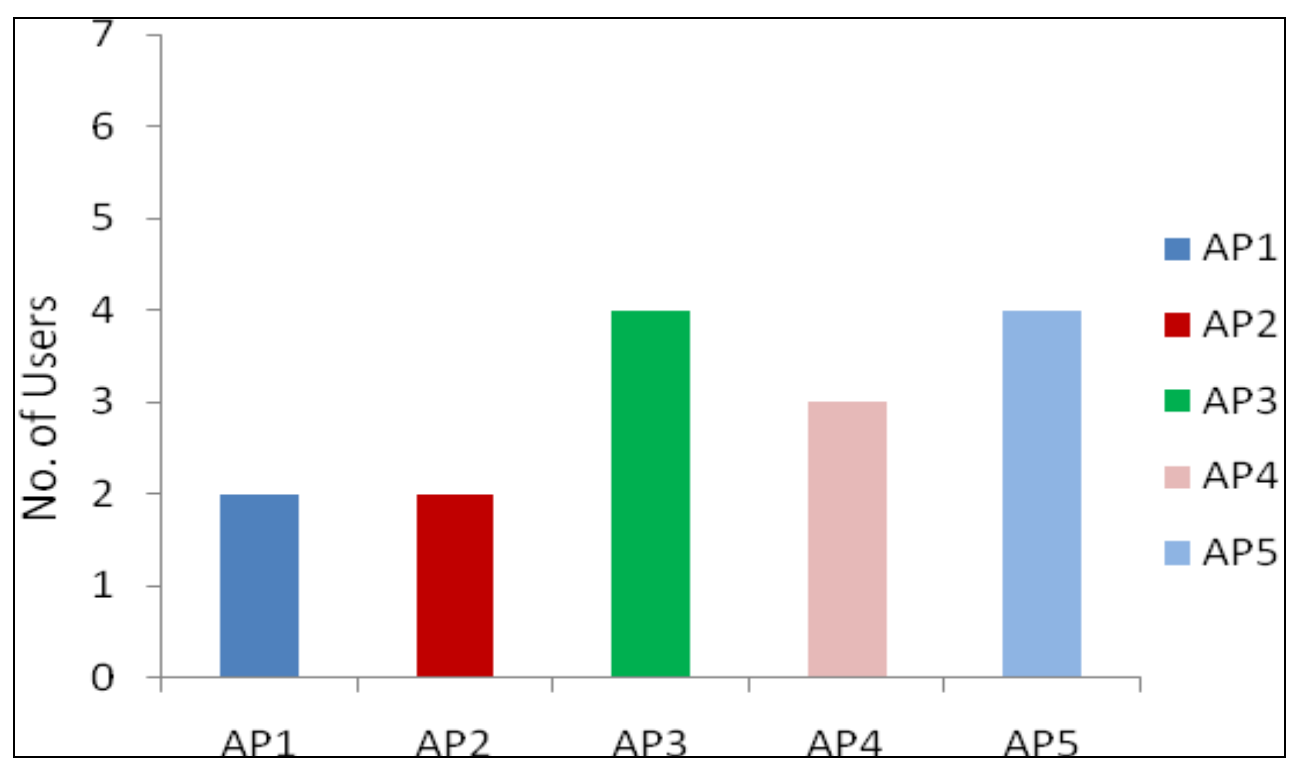

Figure 4(b). Finial User AP Association

From the above result we again notice the improvement in the user traffic by distributing load on different APs. Users of AP1 and AP3 are switched to AP2 and AP5 respectively.

\subsection{Simulation Scenario 3}

In Scenario 3, finally we apply our algorithm on 5 APs and 20 users. This time we have increased the users in network and Comparison results are recorded in figure 5 (b). In this case our 
algorithm gives the best results from all above scenarios by equally distributing user on different APs.

\subsubsection{Range Set}

Range Set of U1 (AP1, AP2, AP4)

Range Set of U2 (AP1, AP2)

Range Set of U3 (AP3, AP4)

Range Set of U4 (AP2, AP1)

Range Set of U5 (AP1, AP2)

Range Set of U6 (AP2, AP4)

Range Set of U7 (AP3, AP4)

Range Set of U8 (AP2, AP4)

Range Set of U9 (AP1)

Range Set of U10 (AP1)
Range Set of U11 (AP1)

Range Set of U12 (AP3)

Range Set of U13 (AP3)

Range Set of U14 (AP3)

Range Set of U15 (AP3)

Range Set of U16 (AP1)

Range Set of U17 (AP3)

Range Set of U18 (AP3)

Range Set of U19 (AP3)

Range Set of U20 (AP3)

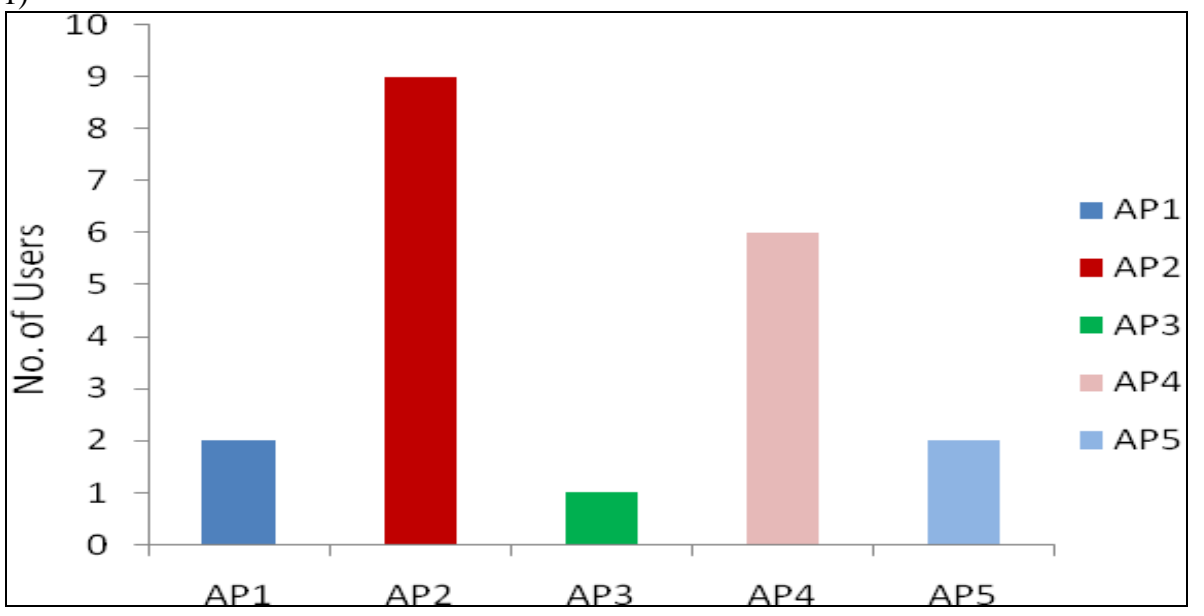

Figure 5(a). Initial User AP Association

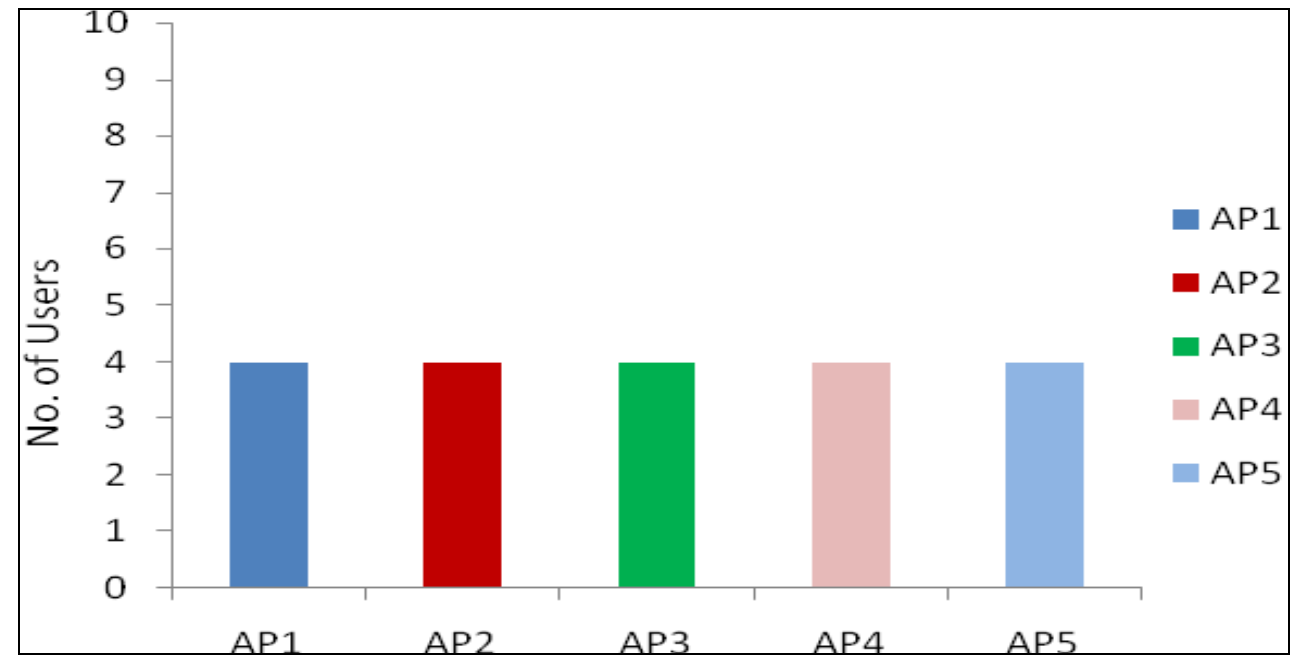

Figure 5(b). Finial User AP Association

We can notice from the above scenarios that our algorithm results are better and efficient in distributing users on different APs. In all scenarios our algorithm shows the significant improvement of load distribution and efficient channel management. 


\section{CONCLUSION}

In this paper, a channel management algorithm has been proposed based on maximizing the channel efficiency and network throughput. Proposed algorithm extends the research to control the network traffic by applying load balancing with moving users in network. Switching of users in network is done after a time slice to manage the load on channels. applications. In future research we can extend our work for outdoor WLAN network.

\begin{tabular}{|c|c|c|c|c|c|c|}
\hline \multirow{2}{*}{\multicolumn{2}{|c|}{ SIMULATION RESULTS }} & \multicolumn{5}{|c|}{ USER TRAFFIC ON DIFFERENT APs } \\
\hline & & \multirow{2}{*}{$\frac{\text { AP } 1}{4}$} & \multirow{2}{*}{$\begin{array}{c}\text { AP } 2 \\
0\end{array}$} & \multirow{2}{*}{$\begin{array}{c}\text { AP } 3 \\
0\end{array}$} & \multirow{2}{*}{$\begin{array}{c}\mathrm{AP} 4 \\
1\end{array}$} & \multirow{2}{*}{$\frac{\text { AP 5 }}{0}$} \\
\hline SCENARIO 1 & INTIAL & & & & & \\
\hline 5 USERS & FINAL & 2 & 2 & $\mathbf{0}$ & 1 & $\mathbf{0}$ \\
\hline \multirow{2}{*}{$\begin{array}{c}\text { SCENARIO } 2 \\
15 \text { USERS }\end{array}$} & INTIAL & 4 & $\mathbf{0}$ & 6 & 3 & 2 \\
\hline & FINAL & 3 & 2 & 4 & 3 & 3 \\
\hline \multirow{3}{*}{$\begin{array}{c}\text { SCENARIO } 3 \\
25 \text { USERS }\end{array}$} & INTIAL & 2 & 10 & 1 & 7 & 5 \\
\hline & FINAL & 5 & 5 & 5 & 5 & 5 \\
\hline & FINAL & 10 & 10 & 10 & 10 & 10 \\
\hline
\end{tabular}

Table 1. Conclusion Result of Different Scenarios

\section{REFERENCES}

[1] Y. Lee, K. Kim, and Y. Choi., Optimization of AP placement and Channel Assignment in Wireless LANs, 27th Annual IEEE Conference on Local Computer Networks, Washington D.C. USA, November 2002, pp. 831-836.

[2] T. Rappaport, Wireless Communications:Principle and Practice, Prentice Hall, pp 19- 23, 2006.

[3] Jim Geier, "Assigning 802.11b access point channels" WiFi Planet,2004.

[4] William Stallings, Wireless Communications \& Networks, Prentice Hall, pp 430, 2005.

[5] M. Haidar, R. Akl, H. Al-Rizzo, Y. Chan, R.Adada, "Optimal Load Distribution in Large Scale WLAN Networks Utilizing a Power Management Algorithm," Proceedings of IEEE Sarnoff Symposium, May 2007.

[6] Channel Assignment In An IEEE 802.11 Wlan Based On Signal-To-interference Ratio Mohamad Haidar, Rabindra Ghimire, Hussain Al-Rizzo, Robert Akl, Yupo Chan, June 2006.

[7] User-based channel Assignment Algorithm in a Loadbalanced IEEE 802.11 WLAN, Mohamad Haidar, Hussain Al-Rizzo, Yupo Chan, Robert Akl.

Table 1 shows the results of various scenarios before and after implementation of the algorithm. The proposed algorithm shows the better results as compared to previous work where users and AP association is fixed. The problem discussed in this paper is developed for research development purposes and also applicable for real-time
[8] Yigal Bejerano, Seung-Jae Han, and Li (Erran) Li, "Fairness and load balancing in wireless lans using association control", Proceedings of ACM Mobicom, 2004.

[9] Anurag Kumar and Vinod Kumar, Optimal Association of Stations and (APs) in an IEEE 802.11 WLAN, Indian Institute of Science, Bangalore, National Communications Conference (NCC), January 2005.

[10] Arunesh Mishra, Vivek Shrivastava, Dheeraj Agarwal,Suman Banerjee, Samrat Ganguly, Distributed Channel Management in Uncoordinated Wireless Environment, MobiCom'06, ACM 1595932860/ 06/0009, Los Angeles, California, USA. September 23-26, 2006.

[11] Ryoichi Sato and Hiroshi Shirai1, SimplifiedAnalysis for Indoor Propagation of a WLAN Channel, 2001 IEEE APS Intl. Symp. Digest, vol.3, July 2001.

[12] Liu Yanbing, Zhai Congcong, Sun Shixin, ANovel Algorithm of Channel Resource Allocation in IEEE 802.11 WLAN, IFIPInternational Conference on Network and Parallel Computing - Workshops, 2007.

[13] Hussain Al-Rizzo, Mohamad Haidar, Yupo Chan, Robert Akl, User-based channel Assignment Algorithm in a Load-balanced IEEE 802.11 WLAN, 66 International Journal of Interdisciplinary Telecommunications and Networking, 1(2), 66-81, April-June 2009.

[14] Amit P. Jardosh Krishna N. Ramachandran Kevin C.Almeroth Elizabeth M. Belding-Royer, Understanding Congestion in IEEE $802.11 \mathrm{~b}$ Wireless Networks, Proceedings of IEEE ASWN, Boston, MA, August 2004. 